\title{
Persistence of Cholinesterase Inhibitor Treatment in Dementia: Insights from a Naturalistic Study
}

\author{
Javier Olazarán ${ }^{a, b} \quad$ Eloísa Navarroc José Manuel Rojo ${ }^{d}$ \\ ${ }^{a}$ Hermanos Sangro Specialties Clinic, Service of Neurology, Gregorio Marañón University \\ Hospital, ${ }^{b}$ Alzheimer Disease Research Unit, Alzheimer Center Reina Sofía Foundation, CIEN \\ Foundation, Carlos III Institute of Health, ' Vicente Soldevilla Specialties Clinic, Service of \\ Neurology, Infanta Leonor Hospital, and ' ${ }^{\mathrm{S}}$ Superior Council of Scientific Research, Madrid, Spain
}

Key Words

Alzheimer's disease · Cholinesterase inhibitors · Lewy body dementia $\cdot$ Treatment persistence

\section{Abstract}

Background: Cholinesterase inhibitors (ChEI) are widely used in dementia, but there is a lack of practice guidelines in case of intolerance or absence of perceived effect. Methods: Two hundred and forty patients (mean age 77 years, SD 6.3, 66\% female) with Alzheimer's disease or Lewy body dementia were prescribed a ChEI and evaluated annually under conditions of standard practice. Of these, 152 patients maintained, 36 switched, and 52 abandoned ChEI treatment. Results: Less behavioural disturbance and less cognitive deterioration were observed, respectively, at the 3- and 4-year follow-up assessments in the patients who maintained the first prescribed ChEI $(p<0.05)$. Cognitive benefits were reinforced in the patients who experienced some adverse event, but no benefits were observed when the patient or caregiver did not perceive an effect. Conclusions: Maintenance of the first prescribed ChEI was supported when some benefit was perceived by the patient or caregiver, even in cases of nonserious adverse events.

Copyright (C) 2013 S. Karger AG, Basel

\section{Introduction}

Randomized controlled trials (RCT) of cholinesterase inhibitors (ChEI) demonstrated small but sufficient efficacy, leading them to become accepted as the primary pharmacological treatment of Alzheimer's disease (AD) - with or without associated cerebrovascular disease - and Lewy body dementia [1, 2]. However, most RCT were of short duration (i.e. 
Olazarán et al.: Persistence of Cholinesterase Inhibitor Treatment in Dementia:

Insights from a Naturalistic Study

12-24 weeks) and, after two decades of widespread use, there are still important unsolved questions regarding long-term efficacy and safety of these medications. In addition, the results obtained from RCT should not necessarily be generalized for standard clinical practice [3].

The conditions of standard practice are quite different from RCT. In standard practice, patients with comorbidity, physical frailty, and combined/less certain aetiologies are more frequent. In addition, physicians choose medications that best match patient and caregiver characteristics, with considerable space for personal experience and preferences [4]. Regarding ChEI, a potentially relevant question, which is clearly open to the physician's subjectivity, is the issue of whether to switch or stay on the first prescribed ChEI in case of intolerance or absence of a clinically apparent effect. In a scenario of nonserious intolerance and good physical health, a trial with a second ChEI seems reasonable. However, when there is good tolerability but lack of perceived effect, continuation, discontinuation, and switch seem equally arguable. Clearly, more research is needed to support such a decision given the potential consequences in terms of safety, efficacy, and cost.

We conducted a naturalistic study (i.e. the ChEI Study) to primarily describe and compare the tolerability of, and the response to, the three most used ChEI (i.e. donepezil, rivastigmine, and galantamine) in patients with cognitive deterioration of any aetiology. In the present article, we focused on data regarding persistence and change of ChEI in those patients with a final diagnosis of AD or Lewy body dementia. The aim was to describe the circumstances associated with (and gain insight on the potential consequences of) maintaining, switching, or abandoning ChEI treatment in those patients.

\section{Methods}

\section{Setting and Study Process}

The ChEI Study was designed and conducted in two neurology clinics that serve two lowmiddle class areas in the southern suburbs of Madrid. Most patients were referred by their family physicians. Patients were systematically and prospectively recruited from January 1 , 2002 to May 31, 2006 by the authors, two senior neurologists with special dedication to dementia. At the beginning of recruitment, the three most used ChEI (i.e. donepezil, rivastigmine, and galantamine) were available (galantamine, the last ChEI to appear, was launched in Spain on September 10, 2001). The inclusion criteria of the ChEI Study were the following: (1) the patient attended the clinic accompanied by a reliable caregiver, (2) cognitive impairment of any aetiology was diagnosed and documented, (3) a ChEI was prescribed for the first time to that patient by the study neurologist, and (4) a 1-year follow-up visit was performed.

All the included patients received a complete medical history that comprised medical history, physical and neurological exam, Mini-Mental State Examination (MMSE) [5], and Clinical Dementia Rating Scale (CDR) [6]. That visit was considered the baseline visit for the ChEI Study. When not available, a structural brain imaging study (i.e. CT scan or MRI) and blood determinations (including blood count, glucose, creatinine, transaminases, calcium, thyroid stimulating hormone, $\mathrm{B}_{12}$, and folate) were ordered [7]. ChEI was prescribed at the baseline visit or 1-3 months later (when tests were ordered). Since the inclusion day was the day of ChEI prescription and a 1-year follow-up visit was required for inclusion, baseline data were retrospectively collected.

ChEI were prescribed according to patient and caregiver characteristics. Due to the simplest titration, donepezil was chosen when there were not many assurances that the patient and the caregiver would comply with the prescribed medication. Rivastigmine was 
Olazarán et al.: Persistence of Cholinesterase Inhibitor Treatment in Dementia:

Insights from a Naturalistic Study

preferred in case of parkinsonism, liver disease, or a high risk of drug interactions. Impaired sleep-wake rhythm and mixed dementia were reasons to choose galantamine [4]. In case of tolerance and good health, the maximum dose of ChEI was tried, usually within a period of time longer than the period recommended by manufacturers. Yearly follow-up visits were scheduled plus additional visits as the clinical situation indicated. Dementia diagnosis and aetiology were established at the baseline visit and those diagnoses were reconsidered at every annual visit.

The present study was designed once the database of the ChEI Study was completed and closed. For the present study, only those patients with a final diagnosis of AD [8] or Lewy body dementia [9], in the presence or not of a vascular component, were selected. Since all the procedures were in accordance with standard practice, no study-specific consent was required. The present study was approved by the ethics committee of Gregorio Marañon University Hospital.

\section{Outcome Variables}

The variables listed below, which were conducted at baseline and annually by the study neurologists during standard practice, were utilized as outcome variables.

Mini-Mental State Examination. This test offers a feasible and universal measure of general cognition for standard practice, with scores ranging from 0 (worse cognitive state) to 30 (best cognitive state) [5].

Functional Assessment Staging Scale. This instrument depicts the predictable functional losses of a typical AD patient [10]. It has been used previously as a measure of functional response to drug treatment in $\mathrm{AD}$ [11]. The possible score ranges from 1 (lack of functional symptoms) to 16 (vegetative state).

Behavioural and Psychological Symptoms of Dementia. During visits, the neurologist elicited information from both the patient and caregiver regarding the following potential behavioural and psychological symptoms of dementia (BPSD): delusions, hallucinations, aggressive behaviour, dysphoria, anxiety, apathy, irritability, aberrant motor behaviour, appetite and eating disorders, euphoria, and disinhibition. Those BPSD categories and their corresponding definitions were taken from the Neuropsychiatric Inventory [12], although this instrument was not formally administered. The BPSD were codified as present or absent. Symptoms were present if they appeared any time from the beginning of cognitive symptoms (baseline visit) or from the last visit (annual follow-up visits). Those BPSD that were completely controlled with medication were codified as absent. Both the total number of BPSD and frequency of the different BPSD were analysed.

Perceived Effect. This was defined as cognitive, functional, affective, or behavioural stabilization or improvement, either transitorily or permanently, as referred to by the patient or caregiver during the study follow-up visits. The effect was usually referred to in the first 1-3 months after ChEI initiation or dose increase.

Safety and Tolerability Measures. All the potential events related to tolerability and safety of the ChEI were requested and registered at every follow-up visit. Caregivers were specifically asked about number of falls, falls with fractures, episodes of loss of consciousness, use of medications, emergency room visits, and hospitalizations.

\section{Statistical Analysis}

Descriptive statistics were used to present patient and caregiver demographic and clinical characteristics and also to present tolerability and safety data. Treatment persistence, change, and abandonment were defined according to the longitudinal data available at the last visit. Persistence on ChEI was defined as maintenance of the initially prescribed ChEI throughout the study period, with neither gaps nor changes of treatment. Change of ChEI was 
Olazarán et al.: Persistence of Cholinesterase Inhibitor Treatment in Dementia:

Insights from a Naturalistic Study

defined as a switch to another ChEI, and abandonment was defined as a lack of any ChEI at the last available visit, independent of ChEI change.

Patient and caregiver differences according to study completion, first prescribed ChEI, and ChEI persistence were analysed at study inception by means of $t$ test and $\chi^{2}$ test. The evolution of cognitive, functional, and behavioural outcomes in patients who persisted on first ChEI, in patients who were switched, and in patients who abandoned ChEI treatment were analysed using repeated measures analysis of covariance adjusted for age and number of chronic diseases. Separate models were elaborated for every annual follow-up assessment, using the baseline assessment as the reference. Analyses were conducted for the complete sample of patients and also, separately, for the subsamples of patients who experienced some adverse event and for whom treatment effect was not perceived (adverse events and perceived effect were referred to the first prescribed ChEI only). To mitigate the effect of study losses, both observed cases and last observation carried forward (LOCF) analyses were systematically conducted. The MMSE, Functional Assessment Staging Scale, and number of BPSD were considered primary outcomes. Because of the potential relevance for clinical practice, post hoc analyses were conducted in the complete sample to ascertain which behavioural domains benefitted from ChEI persistence and the possible repercussion of those behavioural benefits for antipsychotic prescription. Since this was an exploratory study with no previously established hypotheses, multiple comparison adjustment was not conducted [13]. Statistical analyses were performed using SPSS version 16.0 software (SPSS, Chicago, Ill., USA).

\section{Results}

The ChEI Study cohort comprised 270 patients, of whom 240 (89\%) met inclusion criteria for the present investigation. Reasons for exclusion were: no cognitive impairment (1 patient), vascular dementia (4 patients), degenerative aetiology different from AD or Lewy body disease (3 patients), secondary dementia (6 patients), and uncertain aetiology for cognitive impairment or dementia (16 patients). At the baseline assessment, 3 of the 240 included patients (1\%) were institutionalized. There was important attrition throughout the study. As per inclusion criteria, all the patients were evaluated at the 1-year follow-up visit. In addition, 230 patients (95.8\%), 194 patients $(80.8 \%), 161$ patients $(67.1 \%)$, and 136 patients $(56.7 \%)$ could be evaluated in at least one primary outcome measure at the 2-, 3-, 4-, and 5-year follow-up visits, respectively. Patients who did not complete the 5-year follow-up visit tended to be older (mean age 78.1 years, SD 6.8 vs. 76.5 years, SD 6.8, $\mathrm{p}=0.061$ ), tended to be more frequently diagnosed with Lewy body dementia ( 16.3 vs. $8.8 \%, p=0.076)$, and received neuroleptic treatment at the baseline assessment more frequently (9.7 vs. $2.9 \%, \mathrm{p}=0.0278$; all other $\mathrm{p}$ values for differences between noncompleters and completers $>0.10$, data not shown). Persistence, change, or abandonment of ChEI treatment did not influence study loss or death (table 1).

The mean age of the total sample was 77.0 years (SD 6.3, range 51-89) and 66\% of the patients were female. The educational achievement was as follows: illiterate $(16 \%)$, incomplete primary studies (45\%), primary studies (34\%), and superior studies (4\%). Most patients were in the mild stage of dementia according to the CDR: CDR 0.5 (21\%), CDR 1 (69\%), CDR 2 (8\%), and CDR 3 (2\%). Caregivers were more frequently female (70\%) and lived with the patient (68\%). The family link was as follows: daughter or son (50\%), spouse $(40 \%)$, sister or brother (4\%), and other (6\%).

Donepezil was the most frequently first prescribed ChEI $(49.2 \%)$ followed by galantamine $(28.3 \%)$ and rivastigmine $(22.5 \%)$. Donepezil was more frequently prescribed for patients with probable $\mathrm{AD}$, whereas galantamine and rivastigmine were more frequently 
Table 1. Patient attrition in the three study groups

\begin{tabular}{|c|c|c|c|c|}
\hline & $\begin{array}{l}\text { Persisted on first } \\
\text { prescribed ChEI } \\
(n=152)\end{array}$ & $\begin{array}{l}\text { Changed to } \\
\text { other ChEI } \\
(n=36)\end{array}$ & $\begin{array}{l}\text { Abandoned } \\
\text { ChEI treatment } \\
(n=52)\end{array}$ & $\mathrm{p}^{2}$ \\
\hline \multicolumn{5}{|l|}{ 1-year follow-up visit } \\
\hline Died & 0 & 0 & 0 & NA \\
\hline Lost visit & 0 & 0 & 0 & NA \\
\hline Died or lost visit & 0 & 0 & 0 & NA \\
\hline \multicolumn{5}{|l|}{ 2-year follow-up visit } \\
\hline Died $^{1}$ & 0 & 0 & 0 & NA \\
\hline Lost visit & $8(5.3)$ & 0 & $2(3.8)$ & 0.361 \\
\hline Died or lost visit & $8(5.3)$ & 0 & $2(3.8)$ & 0.361 \\
\hline \multicolumn{5}{|l|}{ 3-year follow-up visit } \\
\hline Died $^{1}$ & $5(4.0)$ & $1(3.2)$ & $2(4.2)$ & 0.976 \\
\hline Lost visit & 27 (17.8) & $5(13.9)$ & $6(11.5)$ & 0.536 \\
\hline Died or lost visit & $32(21.1)$ & $6(16.7)$ & 8 (15.4) & 0.614 \\
\hline \multicolumn{5}{|l|}{ 4-year follow-up visit } \\
\hline Died $^{1}$ & $10(9.0)$ & $1(3.8)$ & $3(7.0)$ & 0.659 \\
\hline Lost visit & $43(28.3)$ & $10(27.8)$ & $12(23.1)$ & 0.762 \\
\hline Died or lost visit & $53(34.9)$ & $11(30.6)$ & $15(28.8)$ & 0.690 \\
\hline \multicolumn{5}{|l|}{ 5-year follow-up visit } \\
\hline Died $^{1}$ & 17 (16.7) & $1(4.2)$ & $7(20.0)$ & 0.224 \\
\hline Lost visit & $50(32.9)$ & $12(33.3)$ & $17(32.7)$ & 0.998 \\
\hline Died or lost visit & $67(44.1)$ & $13(36.1)$ & $24(46.2)$ & 0.616 \\
\hline
\end{tabular}

Values represent $\mathrm{n}(\%) .{ }^{1}$ Percentages calculated on the basis of patients for whom information of mortality was available (mortality information could be obtained for 7 patients with otherwise lost visits). ${ }^{2} \chi^{2}$ test.

prescribed for, respectively, patients with $\mathrm{AD}$ with a vascular component and patients with Lewy body disease $(p<0.05)$. Galantamine was also more frequently initiated in case of hypertension, whereas donepezil prescription was associated with an inferior educational achievement $(\mathrm{p}<0.05)$. Rivastigmine prescription was associated with more cognitive deterioration, more apathy, and a higher number of physical comorbidities and medications at baseline assessment ( $\mathrm{p}<0.05$, all other $\mathrm{p}$ values for differences between groups according to the first prescribed ChEI $>0.05$, data not shown).

Most patients (63.3\%) maintained treatment with the first prescribed ChEI in the last study visit. Reasons for ChEI switch or abandonment were intolerance (61.6\%), lack of perceived efficacy (26.7\%), both intolerance and lack of efficacy (7.0\%), and other reasons (4.7\%). Patient and caregiver features according to ChEI persistence are presented in table 2. Patients who were switched to other ChEI were younger in comparison to patients who abandoned ChEI treatment. In addition, patients who changed ChEI received antidepressants at baseline assessment more frequently, whereas patients who persisted on the first ChEI displayed white matter changes more frequently $[14](\mathrm{p}<0.05)$.

A response to the first prescribed $\mathrm{ChEI}$ (either stabilization or improvement) was reported by $45.8 \%$ of patients or caregivers, but some intolerance or adverse event was also recorded in $54.0 \%$ of the total sample. Persistence on the first prescribed ChEI was clearly associated with better tolerability and perceived response, whereas bad tolerability seemed to influence the decision of abandoning ChEI, rather than changing ChEI ( 66.0 vs. 55.6\%, p = 0.142; table 3 ).

Less behavioural disturbance and less cognitive deterioration were observed, respectively, at the 3-and 4-year follow-up assessments in those patients who persisted on the first 
Table 2. Patient and caregiver characteristics by ChEI persistence

\begin{tabular}{|c|c|c|c|c|}
\hline & $\begin{array}{l}\text { Persisted on first } \\
\text { prescribed ChEI } \\
(\mathrm{n}=152)\end{array}$ & $\begin{array}{l}\text { Changed to } \\
\text { other ChEI } \\
(\mathrm{n}=36)\end{array}$ & $\begin{array}{l}\text { Abandoned } \\
\text { ChEI treatment } \\
(\mathrm{n}=52)\end{array}$ & $\mathrm{p}^{1}$ \\
\hline Age, years & $77.1(6.5)$ & $74.8(7.2)^{\mathrm{a}}$ & $79.1(5.7)^{\mathrm{a}}$ & 0.008 \\
\hline Females, \% & 63.8 & 63.9 & 73.1 & 0.461 \\
\hline Education, \% & & & & 0.566 \\
\hline Illiterate & 16.7 & 14.3 & 15.4 & \\
\hline Primary (incomplete) & 49.3 & 34.3 & 42.3 & \\
\hline Primary (complete) & 31.3 & 45.7 & 36.5 & \\
\hline Superior education & 2.7 & 5.7 & 5.8 & \\
\hline Hypertension, \% & 54.6 & 52.8 & 46.2 & 0.574 \\
\hline Diabetes, $\%$ & 23.0 & 27.8 & 25.0 & 0.825 \\
\hline Dyslipaemia, \% & 57.2 & 52.8 & 59.6 & 0.815 \\
\hline Ischaemic cardiopathy, \% & 6.6 & 8.3 & 7.7 & 0.917 \\
\hline Cerebrovascular episode & 10.5 & 11.1 & 7.7 & 0.817 \\
\hline Ever smoker, \% & 25.8 & 27.8 & 23.1 & 0.875 \\
\hline Rosen Scale & $1.1(1.3)$ & $1.1(1.3)$ & $1.2(1.7)$ & 0.951 \\
\hline Chronic diseases $^{2}, \mathrm{n}$ & $2.6(1.7)$ & $2.2(1.5)$ & $3.0(1.6)$ & 0.067 \\
\hline Medications $^{2}, \mathrm{n}$ & $3.1(2.3)$ & $2.8(2.0)$ & $3.0(2.2)$ & 0.806 \\
\hline On antidepressants, \% & 21.7 & 38.9 & 11.5 & 0.010 \\
\hline On neuroleptics, \% & 6.0 & 5.6 & 5.8 & 0.995 \\
\hline On anxiolytics/hypnotics, \% & 22.5 & 22.2 & 15.4 & 0.540 \\
\hline On other medications for BPSD, $\%$ & 2.8 & 2.8 & 3.8 & 0.756 \\
\hline Disease duration, years & $2.6(2.2)$ & $2.4(1.9)$ & $2.2(1.8)$ & 0.412 \\
\hline MMSE & $17.3(5.0)$ & $17.1(4.4)$ & $16.2(4.9)$ & 0.356 \\
\hline FAST & $4.1(0.9)$ & $3.9(1.3)$ & $4.1(0.9)$ & 0.670 \\
\hline$\# B P S D$ & $1.7(1.5)$ & $1.8(1.7)$ & $1.5(1.5)$ & 0.704 \\
\hline Delusions, \% & 16.4 & 13.9 & 13.5 & 0.843 \\
\hline Hallucinations, \% & 6.6 & 13.9 & 5.8 & 0.282 \\
\hline Aggressive behaviour, \% & 17.8 & 22.2 & 19.2 & 0.823 \\
\hline Dysphoria, \% & 38.2 & 41.7 & 28.8 & 0.386 \\
\hline Anxiety, \% & 21.7 & 25.0 & 26.9 & 0.721 \\
\hline Euphoria, \% & 0.0 & 0.0 & 0.0 & NA \\
\hline Apathy, \% & 16.4 & 19.4 & 9.6 & 0.384 \\
\hline Disinhibition, \% & 3.3 & 8.3 & 3.8 & 0.392 \\
\hline Irritability, \% & 30.9 & 16.7 & 19.2 & 0.093 \\
\hline Motor hyperactivity, \% & 4.6 & 2.8 & 3.8 & 0.878 \\
\hline Sleep disorder, $\%$ & 11.8 & 13.9 & 19.2 & 0.410 \\
\hline Appetite and eating, $\%$ & 2.6 & 2.8 & 3.8 & 0.903 \\
\hline Leukoaraiosis $^{3}, \%$ & & & & 0.050 \\
\hline No & 70.0 & 88.9 & 82.0 & \\
\hline Mild & 2.7 & 5.6 & 6.0 & \\
\hline Moderate & 25.3 & 5.6 & 10.0 & \\
\hline Intense & 2.0 & 0.0 & 2.0 & \\
\hline Aetiology, \% & & & & 0.320 \\
\hline Probable AD & 52.6 & 55.6 & 51.9 & \\
\hline Possible AD & 3.9 & 8.3 & 7.7 & \\
\hline AD with vascular component & 33.6 & 16.7 & 26.9 & \\
\hline Lewy body disease & 9.9 & 19.4 & 13.5 & \\
\hline$C D R, \%$ & & & & 0.080 \\
\hline Very mild dementia (CDR 0.5) & 17.8 & 41.7 & 17.3 & \\
\hline Mild dementia (CDR 1) & 72.4 & 50.0 & 73.1 & \\
\hline Moderate dementia (CDR 2) & 8.6 & 5.6 & 7.7 & \\
\hline Severe dementia (CDR 3) & 1.3 & 2.8 & 1.9 & \\
\hline
\end{tabular}


Table 2 (continued)

\begin{tabular}{|c|c|c|c|c|}
\hline & $\begin{array}{l}\text { Persisted on first } \\
\text { prescribed ChEI } \\
(n=152)\end{array}$ & $\begin{array}{l}\text { Changed to } \\
\text { other ChEI } \\
(n=36)\end{array}$ & $\begin{array}{l}\text { Abandoned } \\
\text { ChEI treatment } \\
(n=52)\end{array}$ & $\mathrm{p}^{1}$ \\
\hline \multicolumn{5}{|c|}{ Caregiver characteristics, $\%$} \\
\hline Females & 72.2 & 69.4 & 63.5 & 0.496 \\
\hline Relationship & & & & 0.097 \\
\hline Spouse & 41.7 & 47.2 & 32.7 & \\
\hline Son or daughter & 52.3 & 36.1 & 53.8 & \\
\hline Other & 6.0 & 16.7 & 13.5 & \\
\hline Lived with the patient & 66.0 & 77.8 & 67.3 & 0.392 \\
\hline \multicolumn{4}{|l|}{ First prescribed ChEI, \% } & 0.124 \\
\hline Donepezil & 52.0 & 41.7 & 46.2 & \\
\hline Rivastigmine & 20.4 & 16.7 & 32.7 & \\
\hline Galantamine & 27.6 & 41.7 & 21.2 & \\
\hline \multicolumn{5}{|c|}{$\begin{array}{l}\text { Values represent means (SD), unless \% is indicated. \#BPSD = Number of BPSD; FAST = Functional } \\
\text { Assessment Staging Scale [10]. } \\
{ }^{1} \text { Analysis of variance, global test; between-group differences are represented with superscript letters } \\
\text { (p < } 0.05 \text {, Tukey test). }{ }^{2} \text { Dementia and ChEI were not included. }{ }^{3} \text { On CT or MRI, according to [14]. }\end{array}$} \\
\hline
\end{tabular}

Table 3. Safety, tolerability, perceived effect, and reasons of ChEI withdrawal in the three study groups

\begin{tabular}{lllll}
\hline & $\begin{array}{l}\text { Persisted on first } \\
\text { prescribed ChEI } \\
(\mathrm{n}=152)\end{array}$ & $\begin{array}{l}\text { Changed to } \\
\text { other ChEI } \\
(\mathrm{n}=36)\end{array}$ & $\begin{array}{l}\text { Abandoned } \\
\text { ChEI treatment } \\
(\mathrm{n}=52)\end{array}$ & $\mathrm{p}^{1}$ \\
\hline $\begin{array}{l}\text { Adverse event } \\
\text { Serious adverse event }\end{array}$ & 38.8 & 72.2 & 86.3 & 0.000 \\
$\begin{array}{l}\text { Perceived effect } \\
\quad \text { No }\end{array}$ & 5.3 & 2.8 & 13.7 & 0.067 \\
$\quad$ & 41.7 & 77.8 & 74.5 & 0.000 \\
$\quad$ Yes, stabilization & 24.5 & 13.9 & 13.7 & 0.142 \\
$\quad$ Yes, improvement & 33.8 & 8.3 & 11.8 & \\
$\quad$ Adverse event & NA & 55.6 & 66.0 & 20.0 \\
$\quad$ Lack of efficacy & NA & 36.1 & 6.0 & \\
$\quad$ Adverse event and lack of efficacy & NA & 8.3 & 8.0 & \\
$\quad$ Other reason & & 0.0 & & \\
\hline
\end{tabular}

Values represent percentages. ${ }^{1} \chi^{2}$ test.

prescribed ChEI $(\mathrm{p}<0.05)$ and a trend of less functional deterioration was also documented at the 4-year follow-up visit in those patients $(\mathrm{p}=0.057)$, but statistical significance was not attained in the corresponding LOCF analyses (fig. 1). Post hoc analyses were conducted to ascertain which behavioural domains were improved at the 3-year assessment. Statistically significant differences were only found for prevalence of delusions $(10.9,20.0$, and $40.0 \%$, respectively, for those patients who persisted, changed, and abandoned ChEI treatment, $\mathrm{p}<$ 0.0005 ; all other $p$ values $>0.05$, data not shown). To gain further insight on the effect of ChEI persistence on affective and behavioural disturbance, additional post hoc analyses were 
Olazarán et al.: Persistence of Cholinesterase Inhibitor Treatment in Dementia: Insights from a Naturalistic Study

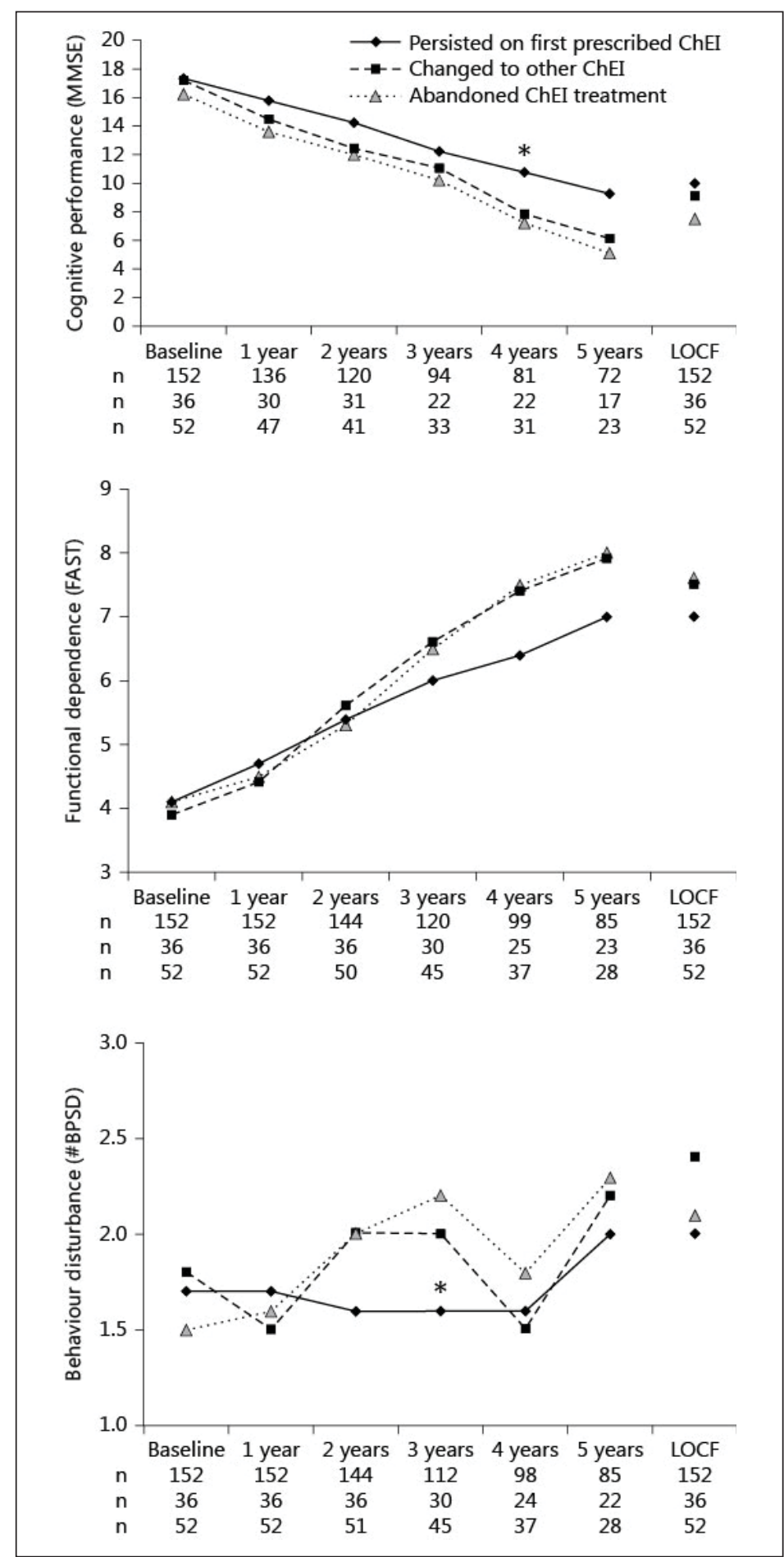

Fig. 1. Cognitive, functional, and behavioural evolution in the three study groups. ${ }^{*} \mathrm{p}=0.014$ (cognitive performance, 4 years) and $\mathrm{p}=$ 0.027 (behaviour disturbance, 3 years) (repeated measures analysis of covariance, baseline score as reference). \#BPSD = Number of BPSD; FAST = Functional Assessment Staging Scale [10].

conducted on the prevalence of psychotropic medication prescription at the 3- and 4-year follow-up visits. A trend of less frequent use of neuroleptics was found at the 3-year followup visit in those patients who persisted on the first prescribed ChEI $(12.7,26.7$, and $24.4 \%$, respectively, for those patients who persisted, changed, or abandoned ChEI treatment; $\mathrm{p}=$ 0.078 ). The corresponding figures at the 4 -year follow-up visit were $13.5,20.0$, and $21.1 \%$ 
Fig. 2. Cognitive, functional, and behavioural evolution in the subsample of patients who experienced some adverse event with the first prescribed ChEI. ${ }^{*} \mathrm{p}=$ 0.005 (cognitive performance, 4 years) and $p=0.007$ (cognitive performance, 5 years) (repeated measures analysis of covariance, baseline score as reference). \#BPSD = Number of BPSD; FAST = Functional Assessment Staging Scale [10].

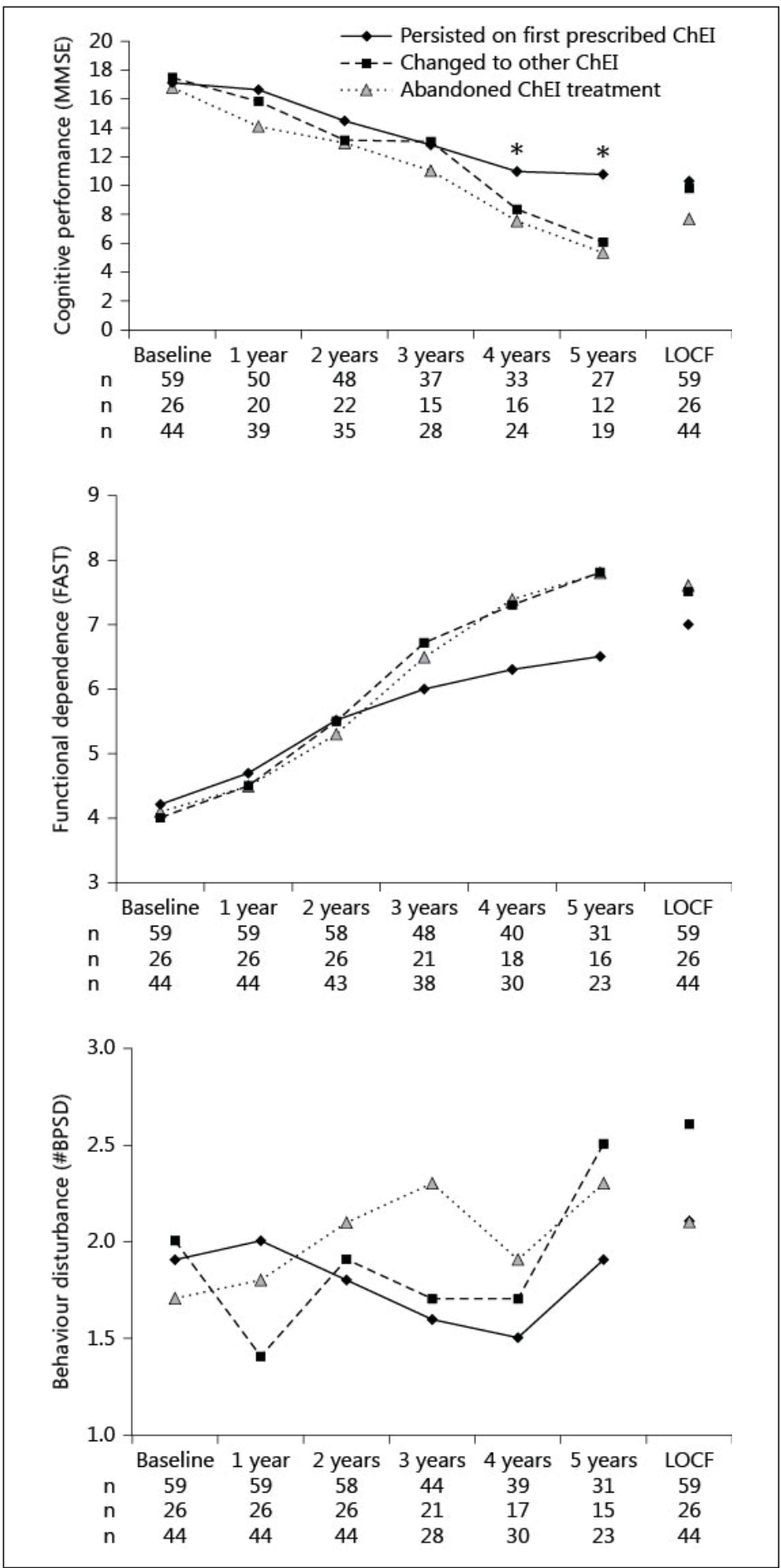

( $p=0.494)$. Other differences or trends in use of psychotropic medications were not found (all $\mathrm{p}$ values $>0.10$, data not shown).

Cognitive and functional results in the subsample of patients who experienced some adverse event were similar to the results observed in the complete sample, with even slightly larger long-term benefits in the patients who persisted on the first prescribed ChEI (fig. 2), but 
Olazarán et al.: Persistence of Cholinesterase Inhibitor Treatment in Dementia: Insights from a Naturalistic Study

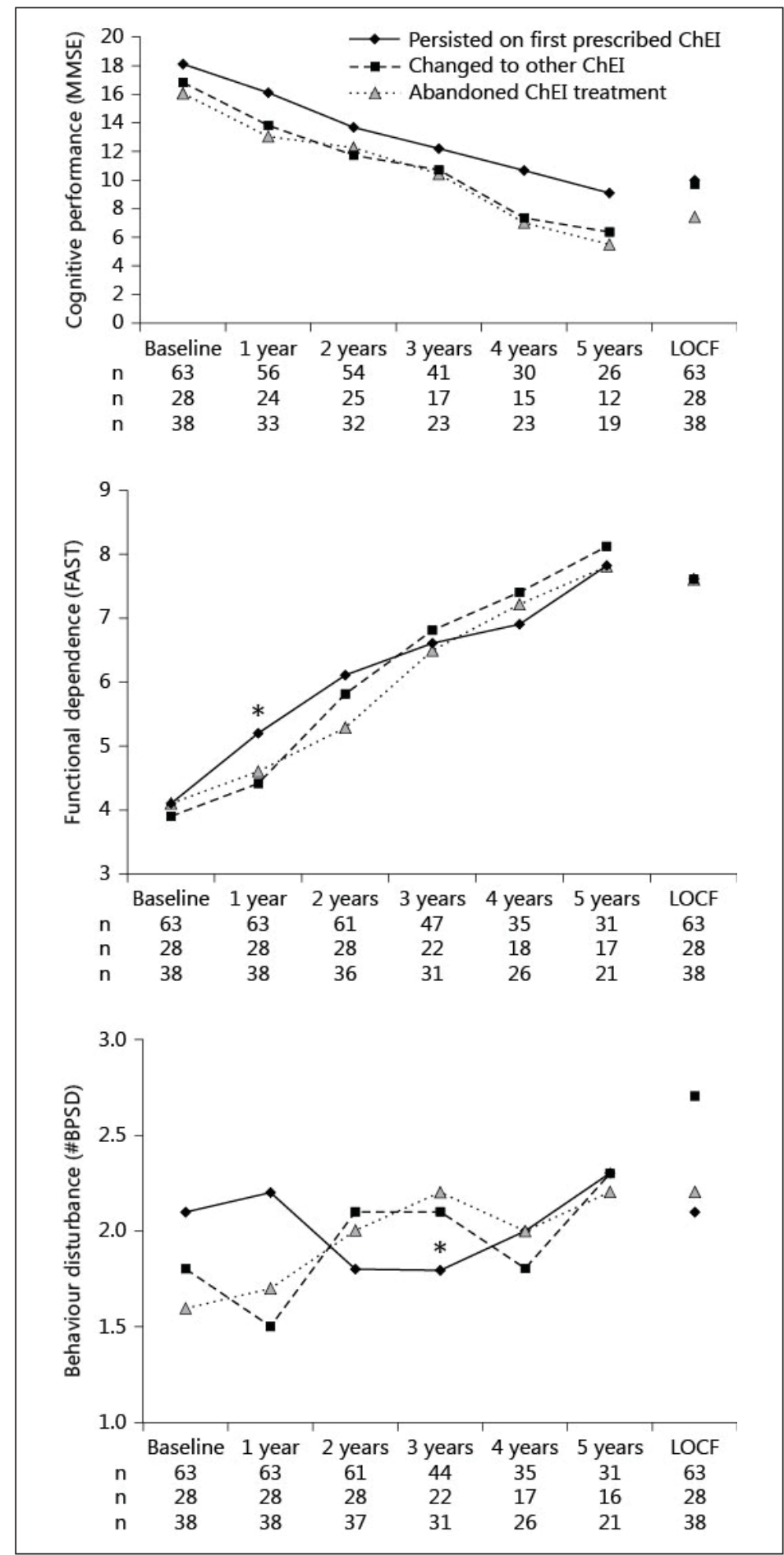

Fig. 3. Cognitive, functional, and behavioural evolution in the subsample of patients who did not perceive a positive effect with the first prescribed ChEI. * $\mathrm{p}=0.025$ (functional dependence, 1 year) and $\mathrm{p}=0.044$ (behavioural disturbance, 3 years) (repeated measures analysis of covariance, baseline score as reference). \#BPSD = Number of BPSD; FAST $=$ Functional Assessment Staging Scale [10].

between-group differences were not detected regarding behavioural evolution. As for the subsample of patients who had no perceived ChEI treatment effect, worse functional evolution was detected at the 1-year follow-up visit in those patients who persisted on the first ChEI, but less BPSD were also detected for those patients at the 3-year follow-up visit. The cognitive evolution of these patients was similar, no matter if they maintained, changed, or abandoned ChEI treatment (fig. 3). 


\section{Discussion}

The causes and effects of ChEI treatment persistence were retrospectively explored in the database of a 5-year observational study that was conducted during standard clinical practice. For the study physicians, the primary aim when prescribing a ChEI was to attain the best balance of clinical benefit, tolerability, safety, and caregiver convenience. In that scenario, a more favourable cognitive, functional, and behavioural outcome was observed in those patients who persisted on the first prescribed ChEI. Those results were reinforced in the subsample of patients who experienced ChEI intolerance, but disappeared in the subsample of patients who, according to patient and caregiver, did not experience positive effect.

Treatment persistence did not depend on the prescribed ChEI. In this regard, a trend of more favourable outcome for donepezil was noted (table 2), but it should be said that, at study inception, neither galantamine extended-release capsules nor rivastigmine patches were available. Hence, under quite different patient and caregiver circumstances and once the ChEI that best fit the particular patient and caregiver dyad was chosen, persistence on the different ChEI was similar. This observation reinforces the view that results from clinical trials do not necessarily generalize to clinical practice [15], with an important role left to physicians to optimize ChEI effects.

What other lessons can we extract for standard practice? We believe that physicians should be proactive when discussing ChEI treatment with otherwise healthy AD or Lewy body dementia patients and caregivers, in light of the consistent and durable positive results observed in the domains of cognition, function, and behaviour. In addition, the presence of concomitant cerebrovascular disease, on the basis of clinical or neuroimaging data, should not hinder a proactive attitude towards ChEI treatment. It also seems clear that the first ChEI inhibitor should be maintained if some positive response is perceived, even in case of some intolerance, provided there are no important safety concerns. A titration slower than usual could be of help in such cases.

When important intolerance is experienced during the early titration phase, with no place for evaluating a potential clinical benefit, a trial of a second ChEI seems reasonable after complete resolution of side effects and provided there is no serious risk. However, what should one do when there is no perceived effect once the maximal dose is achieved and well tolerated? A possible response to a second ChEI, after failure of the initial ChEI, has not been extensively or rigorously investigated. Improvement with a second ChEI has been described in open trials, but those results could have been influenced by expectation bias $[16,17]$. The results from the present investigation suggest that a lack of response to ChEI could be a class effect, with little space for trying different ChEI. Identifying predictors of ChEI response remains an important but elusive issue [18]. Patients who did not respond to ChEI could be good candidates for trials of new medications, where no treated patients would be preferable.

The present study has strengths and limitations. A representative sample of patients was administered a ChEI on an individual basis, without financial restrictions, and followed for over 5 years. However, most significant results emerged in the long term, when there was important study attrition. In addition, the study groups were not fully comparable at inception. Patients who abandoned ChEI treatment were older and tended to have more chronic diseases (table 2), and patients who were lost to follow-up also tended to be older. Hence, caution should be taken before generalizing the present results to the physically frail old population.

It could also be speculated that our patients who maintained ChEI presented with a slower cognitive and functional deterioration that influenced the physician's decision of staying on the initial ChEI. This explanation cannot be definitively ruled out given the observational design, but it seems unlikely to the authors for two reasons. First, disease duration and severity of dementia before treatment were comparable in the three study groups 
Olazarán et al.: Persistence of Cholinesterase Inhibitor Treatment in Dementia: Insights from a Naturalistic Study

(table 2). And second, a better response to ChEI was reported in patients with more rapid cognitive deterioration [18].

In conclusion, ChEI seem to be effective and safe for the long-term treatment of Alzheimer's and Lewy body dementia, even in the presence of nonserious adverse events. A simple perception of benefit by the patient or caregiver was a good indicator of objective clinical response and could be of help in the decision of maintaining ChEI treatment or not. Final decisions regarding treatment should always be taken after considering the individual characteristics of the patient and caregiver, including their personal opinions and values.

\section{Disclosure Statement}

Dr. Olazarán has received research support from Eisai. He has also received speaker's honoraria from Grunenthal, Esteve, Pfizer, Novartis, Janssen, and Lündbeck. Dr. Navarro has received speaker's honoraria from Esteve, Pfizer, Novartis, Grunenthal, and Lündbeck. Dr. Rojo declares no conflict of interest.

\section{References}

1 Raina P, Santaguida P, Ismaila A, Patterson C, Cowan D, Levine M, et al: Effectiveness of cholinesterase inhibitors and memantine for treating dementia: evidence review for a clinical practice guideline. Ann Intern Med 2008;148:379-397.

- 2 Howard R, McShane R, Lindesay J, Ritchie C, Baldwin A, Barber R, et al: Donepezil and memantine for moderateto-severe Alzheimer's disease. N Engl J Med 2012;366:893-903.

3 Pavlik VN, Doody RS: Progress in understanding variability in cognitive responses to cholinesterase inhibitor treatment. Alzheimers Res Ther 2011;3:30.

- 4 Gil P, Ayuso JL, Marey JM, Antón M, Quilo CG: Variability in the diagnosis and management of patients with Alzheimer's disease and cerebrovascular disease: results from the GALATEA multicentre, observational study. Clin Drug Investig 2008;28:429-437.

5 Folstein MF, Folstein SE, McHugh PR: Mini-mental state: a practical method for grading the cognitive state of patients for the clinician. J Psychiatr Res 1975;12:189-198.

- 6 Hughes CP, Berg L, Danziger WL, Coben LA, Martin RL: A new clinical scale for the staging of dementia. Br J Psychiatry 1982;140:566-572.

7 Feldman HH, Jacova C, Robillard A, Garcia A, Chow T, Borrie M, et al: Diagnosis and treatment of dementia: 2. Diagnosis. CMAJ 2008;178:825-836.

- 8 McKhann G, Drachman D, Folstein M, Katzman R, Price D, Stadlan EM: Clinical diagnosis of Alzheimer's disease: report of the NINCDS-ADRDA Work Group under the auspices of Department of Health and Human Services Task Force on Alzheimer's Disease. Neurology 1984;34:939-944.

- 9 McKeith IG, Dickson DW, Lowe J, Emre M, O’Brien JT, Feldman H, et al: Diagnosis and management of dementia with Lewy bodies: third report of the DLB Consortium. Neurology 2005;65:1863-1872.

10 Reisberg B: Functional assessment staging (FAST). Psychopharmacol Bull 1988;24:653-659.

-11 Reisberg B, Doody R, Stöffler A, Schmitt F, Ferris S, Möbius HJ, Memantine Study Group: Memantine in moderate-to-severe Alzheimer's disease. N Engl J Med 2003;348:1333-1341.

12 Cummings JL, Mega M, Gray K, Rosenberg-Thompson S, Carusi DA, Gornbein J: The neuropsychiatric inventory: comprehensive assessment of psychopathology in dementia. Neurology 1994;44:2308-2314.

13 Rothman KJ: No adjustments are needed for multiple comparisons. Epidemiology 1990;1:43-46.

14 Wahlund LO, Barkhof F, Fazekas F, Bronge L, Augustin M, Sjögren M, et al: A new rating scale for age-related white matter changes applicable to MRI and CT. Stroke 2001;32:1318-1322.

15 Suh DC, Thomas SK, Valiyeva E, Arcona S, Vo L: Drug persistency of two cholinesterase inhibitors: rivastigmine versus donepezil in elderly patients with Alzheimer's disease. Drugs Aging 2005;22:695-707.

16 Gauthier S, Emre M, Farlow, Bullock R, Grossberg GT, Potkin SG: Strategies for continued successful treatment of Alzheimer's disease: switching cholinesterase inhibitors. Curr Med Res Opin 2003;19:707-714.

17 Massoud F, Desmarais JE, Gauthier S: Switching cholinesterase inhibitors in older adults with dementia. Int Psychogeriatr 2011;23:372-378.

18 Wallin AK, Hansson O, Blennow K, Londos E, Minthon L: Can CSF biomarkers or pre-treatment progression rate predict response to cholinesterase inhibitor treatment in Alzheimer's disease? Int J Geriatr Psychiatry 2009;24:638-647. 